\title{
Functional gradients through the cortex, multisensory integration and scaling laws in brain dynamics
}

\author{
Isabel Gonzalo-Fonrodona \\ Departamento de Óptica. Facultad de Ciencias Físicas. \\ Universidad Complutense de Madrid. Ciudad Universitaria s/n. 28040-Madrid. Spain \\ E-mail: igonzalo@fis.ucm.es
}

\begin{abstract}
In the context of the increasing number of works on multisensory and cross-modal effects in cerebral processing, a review is made on the functional model of human brain proposed by Justo Gonzalo (1910-1986), in relation to what he called central syndrome (caused by unilateral lesion in the parieto-occipital cortex, equidistant from the visual, tactile and auditory projection areas). The syndrome is featured by a bilateral, symmetric and multisensory involvement, and by a functional depression with dynamic effects dependent on the neural mass lost and related to physiological laws of nervous excitability. Inverted or tilted vision as well as tactile and auditive inversion, under minimum stimulus, appears as a stage of incomplete integration, being almost corrected under higher stimulus or facilitation by multisensory integration. The syndrome reveals aspects of the brain dynamics that suggest a functional continuity and unity of the cortex. A functional gradients scheme was proposed in which the specificity of the cortex is distributed with a continuous variation. This syndrome is interpreted as a scale reduction in the nervous excitability of the system, the different sensory qualities being affected allometricaly according to scaling laws. A continuity from lower to higher sensory functions was proposed. The sensory growth by an increase of the stimulus or by multisensory facilitation is found to follow approximately power laws, that would reflect basic laws of biological neural networks. We restrict the analysis to the visual system.
\end{abstract}

Key words: multisensory, cross-modal effects, facilitation, neurophysiology, inverted perception, tilt illusion, visual system, brain dynamics, scaling laws.

\section{INTRODUCTION}

In recent years, many works reported on multisensory and cross-modal effects in integrative cerebral processes (e.g., 1, 2, 3, 4, 5, 6, 7, 8, 9, 10, 11, 12] referring only to some recent works among many others). It was found that cross-modal interactions can affect activity in cortical regions traditionally regarded as "unimodal", as it occurs for example in the contribution of visual cortex to tactile perception 11, 13, 14]. Some of these multisensory interactions have been revealed by functional magnetic resonance imaging, positron emission tomography and analysis of blood oxygen level. According to some authors, all these findings suggest that longstanding notions of cortical organization need to be revised to include multisensory interactions as a inherent component of functional brain organization [1] (see also [15] and references therein).

In this order of ideas, we recall in the present work the pioneering research of Justo Gonzalo (1910-1986) [16, [17, 18]. This author studied about one hundred selected patients with brain injuries from the Spanish Civil War (1936-39). Twelve of them with brain injury in the parieto-occipital zone and special features led to characterize what the author called "central"syndrome of the cortex, and to propose a dynamic interpretation of cerebral localizations. This allowed the author to explain the cases of first hand and others reported in the literature as the much-discussed Schn. case [19]. The author developed a functional model of some aspects of brain dynamics based on functional gradients through the cortex and sca- ling laws of dynamic systems, supported by physiological laws of nervous excitability. It was exposed in part in 16] and further developed in $17,18,20,21,22,23,24,25,26]$. It offers a framework to understand at a functional level, several aspects of the integrative cerebral process. The immediate repercussion of this research (e.g., 27, 28, 29]) diverted later towards the development of cerebral processing models [23, 24, 30, 31, 32, 33], apart of historical notes [34, 35].

The central syndrome [16, 17, 18] is originated from a unilateral parieto-occipital lesion equidistant from the visual, tactile and auditory projection areas ("central" zone) as shown in Fig.1, A (the middle of area 19, the anterior part of area 18 and the most posterior of area 39, in Brodmann terminology). It presents a multisensory and symmetric affection; visual, tactile and auditory systems are equally affected, in all its functions and with symmetric bilaterality. In the visual system for example, in addition to other disorders, there is a bilateral and symmetric concentric reduction of the visual field (Fig. 1. A) with gradation of the involvement from the center to the periphery. This syndrome involves a loss of rather unspecific (or multispecific) neural mass and is characterized by a functional depression shown for example in the excitation threshold curves in Fig. 22 [16, 18]. In this figure we see that the thresholds of the acute case $M$ are greater than those of the less intense case $\mathrm{T}$ (with a smaller lesion), and the thresholds of $\mathrm{T}$ are greater than in a normal man. This depression presents dynamic phenomena related to transformations of the central nervous excitability which depend on the quantity of neural mass 


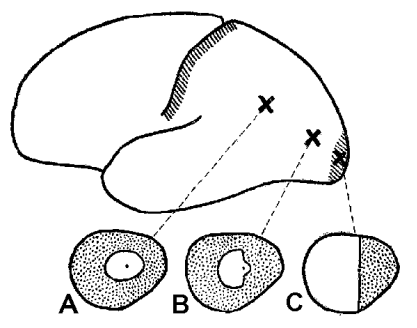

Figura 1: Position of cortical lesions and respective visual fields (involved zones are dark). A: central, B: paracentral, C: marginal or peripheral, syndromes. (Adapted from Fig. 1(a) of [20] with permission of the MIT Press).

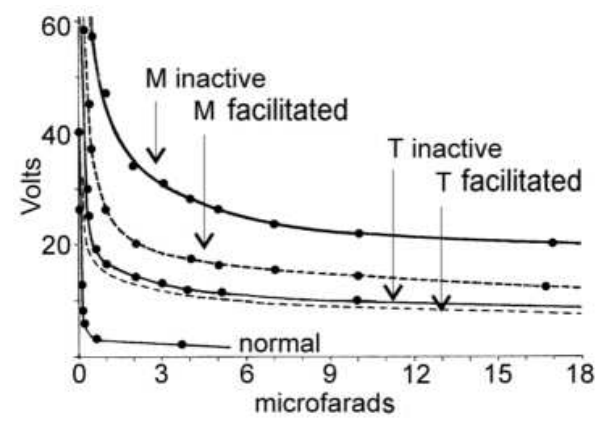

Figura 2: Excitation threshold curves for electrical stimulation of the retina (cathode on eyelid) in acute central syndrome case $\mathrm{M}$, less intense case $\mathrm{T}$, each one in inactive state and facilitated state by strong muscular stress (see the text), and in a normal case. Electrical intensity (indirectly given by volts) versus time (given by microfarads) necessary to obtain minimum luminous sensation. (From Fig. 2 of [25] with permission of Viguera Editores, S.L.).

lost. These phenomena are [16, 18]:

(a) Functional disgregation or dissociation of sensory qualities (normally united in perception) according to their excitability demands, i.e., sensory qualities are gradually lost as the stimulus intensity diminishes. For instance, when the illumination of an upright white arrow was diminishing, the arrow perceived is at first upright and well defined, next it is perceived to be more and more rotated in the frontal plane (Fig. 3) becoming at the same time smaller and losing its form and colors in a well-defined physiological order, the perceived tilt (almost inverted vision in the acute syndrome case $\mathrm{M}$ ) being dependent on the size, distance, illumination and exposure time of the test object. In the tactile system, five stages were distinguished successively in the dissociated perception of a mechanical pressure stimulus on one hand, as the energy of the stimulus was increased: first, primitive tactile sensation without localization, then, contralateral localization (inverted perception), and finally, normal localization which required intense stimulus. A mobile stimulus was perceived in the inversion phase with a very shortened trajectory (approximately $1 / 10$ in case

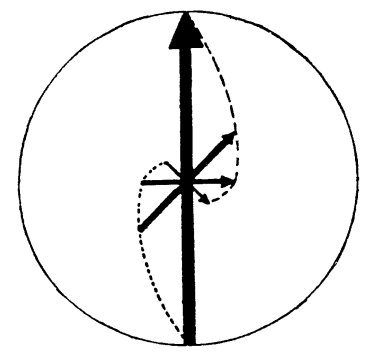

Figura 3: Perceived tilt and diminution in size of a vertical test arrow in the center of the visual field of right eye in central syndrome, as the illumination of the arrow diminishes. Spiral branches described by the extremes of the arrow are indicated. (Adapted from Fig. 3(a) of [20] with permission of Springer Science and Business Media).

M). In the auditory system, there was a dissociation between simple sonorousness (weak stimulus) and real tone (stronger stimulus) of a particular sound. Contralateral localization (spatial inversion) of a sound stimulus occurred only in the most acute case $\mathrm{M}$ when the stimulus was weak and the patient was in inactive state (free of facilitation). The inverted perception always lacked tonal quality. In language, diverse aphasic aspects occurred depending on the stimulation, it will be exposed in a future work.

(b) Capability to multisensory facilitation (the perception of a stimulus is improved by adding simultaneously one or more different stimuli). It modifies the cerebral system essentially, becoming more rapid and excitable, i.e., it supplies in part the neural mass lost in the "central" lesion, thus reducing the mentioned dissociation. It was found that a strong muscular stress was very efficient at improving the perception (see the facilitated cases in Fig. 2. Other types of facilitation to visual perception were binocular summation and tactile and acoustic stimuli, for example.

(c) Capability to temporal summation, which is merely a particular means of stimulation. The slowness of the cerebral system in central syndrome makes the cerebral excitation to a short stimulus to decay slowly. If a second stimulus arrives before this excitation has completely fallen down, excitations are summed up, so that it is possible to achieve an excitation threshold to produce a sensory perception, reducing so the mentioned pathological dissociation. It was shown [23] that it is possible to model the temporal dynamics of simple sensory functions as a first-order linear time-dispersive system having as a first approach a time response $\chi_{0} e^{-a / t}$ ( $t$ is time) to a short impulse stimulus. The reaction velocity $a$ and the permeability to the excitation $\chi_{0}$ of the cerebral system decrease when the deficit (due to the lesion) of active "central" neural mass is greater, but the ratio $a / \chi_{0}$ increases significantly and can be considered as a constant for each sensory function of a given cerebral system without facilitation. 
Thus, the importance of the central syndrome (say, symmetric multisensory syndrome) lies in the fact that changes in the stimulation intensity (and then in nervous excitation) reveal aspects of the dynamics of the cerebral cortex, multisensory integration, and organization of sensory structures combined with the direction function manifested in the tilted or almost inverted perception. Spatial inversion appears as an essential fact in the sensory organization. In a recent review 25], the inverted or tilted perception disorder observed and interpreted by Gonzalo, is put in relation with the increasing number of reported cases with this anomaly in the last years (for example 37, 38, 39, 40, 41, 42] among many others reviewed in [25]). In tilted vision, a rather similar degradation of the perception to that referred in (a) was reported in [42].

We expose in the following the principal features of the model proposed by Gonzalo highlighting the connection with recent research.

\section{FUNCTIONAL CEREBRAL GRADIENTS}

From the first hand cases and others reported in the literature, the diverse syndromes were ordered according to the position and magnitude of the lesion [18]. The central syndrome refers, as said above, to lesions in the "central"zone, equidistant from the visual, tactile and auditive projection paths. Syndromes corresponding to lesions in the projection paths were called in this context "marginal" or "peripheral" syndromes, the defect functional suppression- is restricted to the contralateral half of the corresponding sensory system and scarcely present dynamic effects. Intermediated syndromes were called "paracentral" syndromes, with different degree in the bilateral symmetry involvement (see Fig. 1).

The complete gradation found between the central syndrome, and a marginal syndrome, through the variety of paracentral syndromes lead to the definition of two types of continuous functions through the cortex called "cerebral gradients" [18], shown schematically in Fig. 4, One type comprises the specific sensory functional densities, of contralateral character, with maximum value in the respective projection area and decreasing gradually towards more "central" zone and beyond so that the final decline of the specific visual function density, for example, must reach all the tactile area, as shown in Fig. [4 as well as other specific areas including their primary zones. This type of function takes into account and combines the factors of position and magnitude of the lesion, since the more "central" is the lesion, the more extensive must be the lesion to originate the same intensity in a specific anomaly. For a given position of the lesion, its magnitude determines de degree of functional depression. The other type of function, of unspecific (or multispecific) character, is maximum in the "central" region (where the decline of the above mentioned specific functions overlap) and vanishes towards the projection areas. It re-

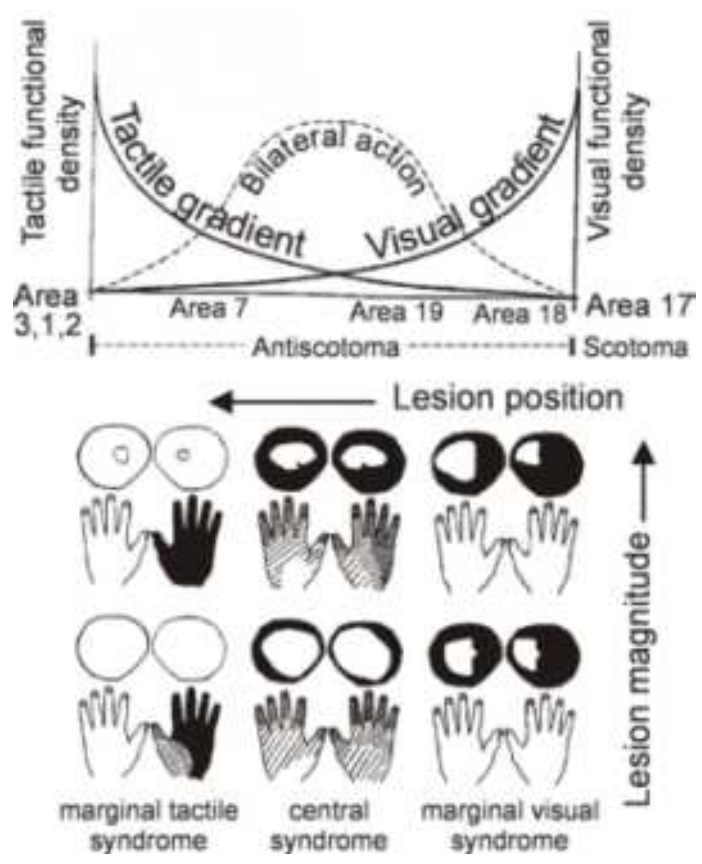

Figura 4: Lower part: visual fields and tactile sensitivity of cases ordered according to the position and magnitude of the lesion. The degree of the defect is greater in darker regions. Upper part: scheme of visual and tactile functional densities $f$, and the unspecific functional gradation which represents the multisensory and bilateral effect. Brodmann areas are indicated. (Adapted from Fig. 5 of [20] with permission of The MIT Press).

presents the multisensory effect in the anomalies and the bilaterality or interhemispheric effect by the action of the corpus callosum. Each point of the cortex is then characterized by a combination of specific contralateral action with unspecific "central" and bilateral action.

In the visual system for example, for the visual function to be normal, the action of the region with greatest visual sensory function density is not enough, and the whole specific functional density, say f, in gradation through the cortex, must be involved in the integrative cerebral process, leading to the normal sensory visual function $F$. Analogously for the other senses and qualities. In a schematic way, $f$ is related to the derivative of this integration through the cortex, which justifies it was named gradient. This function $f$ takes into account the density of specific neurons through the cortex and their connections, representing the dynamic aspect of its anatomic basis. A sensory signal in a projection area is only an inverted and constricted outline that must be elaborated (magnified, reinverted, ...), i.e., integrated over the whole region of the cortex where the corresponding specific sensory functional density $f$ is extended. Magnification would be due to the increase in recruited cerebral mass, and reinversion due to some effect of cerebral plasticity, (following an spiral growth as the growth of the arrow in Fig. (3). In the visual system, reinversion and 


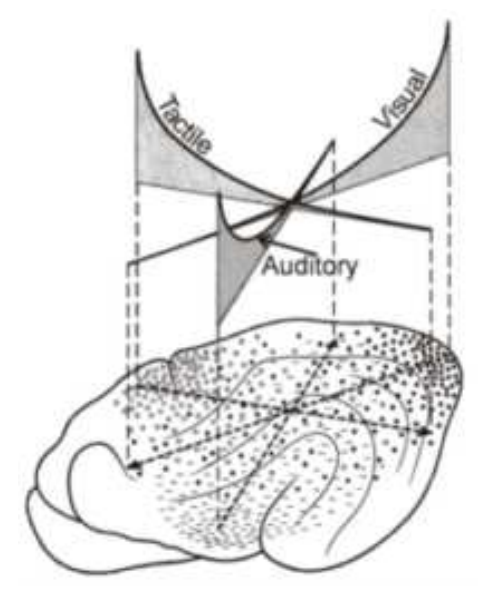

Figura 5: Schematic visual, tactile and auditory functional densities.

bilateralization would occur in the 18 and 19 Brodmann areas where the sensory representation is already reinverted. A remarkable result is, for example, the significant participation of the traditionally "extravisual" cortex in the maintenance of the visual field.

The projection zones where the respective specific functional gradients are maximum, are highly organized and differentiated (in biological terms), i.e., highly specialized. They are phylogenetically the oldest zones of the isocortex and the nervous activity has an anatomic representation. In contrast, the "central" zone which is more recent, is rather unspecific with capacity for adaptation or learning. It is very small in animals, even in other mammals, but it has great extension in man. A schematic representation of the visual, tactile and auditory gradients is shown in Fig. 5 .

Certain sensory functional densities would arise from the superposition of functional gradients of different systems, as in the case of primary alexia where the corresponding functional specific (lexical) gradient would result from the superposition of auditory and visual ones, leading to a lexical bell gradient between the two systems. In some cases, a gradient with hemispherical dominance has to be added.

As opposed to the rigid separation of zones, the functional gradients account for a functional continuity and physiological heterogeneity of the cortex, this one being subjected to a common principle of organization. This scheme, mere abstraction of the observed facts, offers a dynamic conception of quantitative localizations which permits an ordering and an interpretation of multiple phenomena and syndromes. Also, this scheme is in relation to recent works suggesting that traditional specific cortical domains are separated from one another by transitional multisensory zones [15], and that multisensory interactions occur even in the primary sensory cortices $[1,[3,13]$.

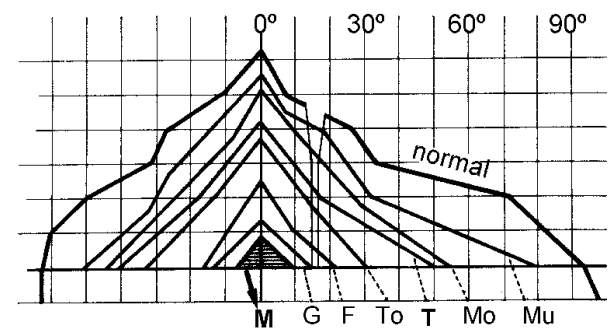

Figura 6: Sensibility profiles of visual fields in different central syndrome cases ( $\mathrm{M}$ and $\mathrm{T}$ included) and in a normal case. (Adapted from Fig. 2 of [21] with permission of Springer Science and Business Media).

\section{SCALE REDUCTION IN CENTRAL SYNDROME. ALLOMETRIC SCALING LAWS IN THE DISPLAY OF SENSORY QUALITIES}

The cerebral system after a "central"lesion, once the new dynamic equilibrium is reached, was considered a scale reduction in the nervous excitability of the cerebral system since the originated functional depression maintains, nevertheless, the same cerebral organization as in normal case. This can be appreciated, for example, in the hypoexcitability of the nervous centers shown in the excitability (Fig. 2) and luminosity threshold curves, and also in the concentric reduction of the visual field, its sensibility profile (see Fig. 6) and visual acuity. All these functions experienced a down shifting in their values, but keeping the same form as in a normal case [18].

Then, the concept of dynamic similitude, according to which different parts of a dynamic system change differently under a change in the size of the system, was applied. In particular, in biological growth, the sizes of two parts (say x and y) of a biological system are approximately related by a scaling power law of the type

$$
y=A x^{n},
$$

$n$ being different for different parts $\left(y_{1}, y_{2}, \ldots\right)$ of the system. These parts change then differently, i.e., allometrically [43]. This power relation means that the rates of growth of the two parts compared are proportional, i.e., $(1 / y)(d y / d t)=n(1 / x)(d x / d t)$ (obtained from Eq. (1) by taking the logarithm and differentiating). These ideas were applied by Gonzalo 21] to the growth (or reduction) of the sensory qualities, linked each one to a nervous excitation demand (i.e., to a quantity of neural mass). An allometric variation of the sensory qualities was then proposed.

For the pure central syndrome cases studied [18], normalized values of the visual direction function $y_{1}$ and of the acuity function $y_{2}$, versus normalized untouched visual field surface $x$, are plotted in Fig. 7 (a). The acute case $\mathrm{M}$ (with considerable neural mass lost), the less intense case $\mathrm{T}$ (with less neural mass lost than $\mathrm{M}$ ) and a normal case $\mathrm{N}$ are indicated. Errors are greater in cases 

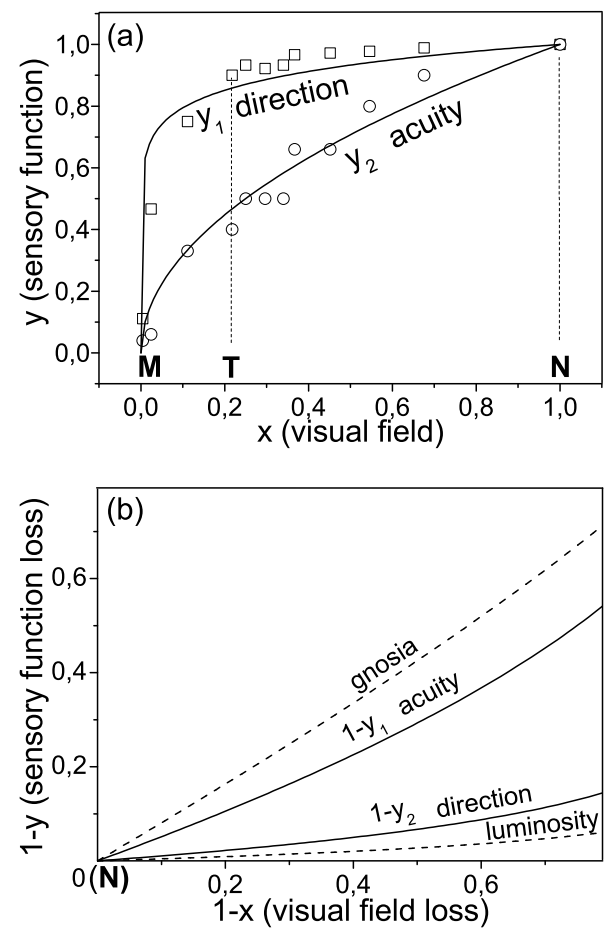

Figura 7: (a)Normalized direction function (squares), fitting to $y_{1}=x^{0,5}$, and normalized acuity function (circles), fitting to $y_{2}=x^{0,1}$, for central vision, versus normalized untouched visual field surface $x$ of the observing eye. Cases M, T and normal $(\mathrm{N})$ are indicated. (b) Curves for the loss of visual acuity, $1-y_{1}$, and loss of direction, $1-y_{2}$, versus loss of the visual field, $1-x$, for the same conditions and normalizations as in (a). Qualitative indications for the loss of the higher sensory function gnosia and lower sensory function luminosity are shown as examples.

with intense central syndrome because of their high sensory degradation and the high capability to facilitation by temporal and multisensory summation. The values plotted are normalized with respect to the normal value. The direction function is considered $0^{\circ}$ for the total inverted perception of the upright test arrow, and $180^{\circ}$ for the upright perception (normal), i.e., the maximum value is achieved in a normal integrative process from the inverted signal in the projection path. For a normal case, N, the normalized values $x, y_{1}$ and $y_{2}$ are the unity, the maximum value. For case $\mathrm{M}$, the corresponding values are very small since the non-normalized values are, 6 degrees for the visual field width, 0.04 for the acuity and 160 degrees for the perceived direction.

We can see in Fig. 7(a) that the data fit approximately to power laws. Since $y_{1}$ and $y_{2}$ are 1 for $x=1$, the value $A$ of Eq. (1) is 1 and the approximate allometric power laws found are $y_{1}=x^{0,5}$ for the direction function and $y_{2}=x^{0,1}$ for the acuity function. If the widths of the visual fields are considered instead of the surface values $x$, there is no evidence of power laws.

In order to appreciate the loss of the sensory functions in the pathological disgregation of the sensorium in central syndrome, the loss of direction function $\left(1-y_{1}\right)$ and the loss of visual acuity $\left(1-y_{2}\right)$ are plotted in Fig. $7(\mathrm{~b})$ versus the loss of visual field $(1-x)$, together with a qualitative representation of the loss of other functions: an elementary one as luminosity and a higher one as gnosia, according to the observed facts. The origin of the graphs corresponds to a normal man $(\mathrm{N})$. We can see that for a particular loss of visual field (due to the scale reduction originated by a particular central lesion), a split of the different qualities occurs so that the higher ones (e.g., gnosia) are loss in a higher degree than the lower (elementary) ones (e.g., luminosity). The order of the split corresponds to the order of complexity (or excitability demand) of the sensory functions and to the order they are lost due to the shifts in their threshold excitabilities.

This is the formal description of the mentioned functional depression where the most complex qualities, with greatest nervous excitability (and integration) demand, become lost or delayed in greater degree than the most simple ones (with lower excitability demand). Sensations usually considered as elementary are then seen to be decomposed into several functions, one of them being the direction function, thus revelling up to a certain extent, the organization of the sensorium. Very small differences in the excitation of different qualities occur already in the normal individual (in colors for example), and they grow considerably in central syndrome. In this context, it could be said that the cerebral system of the normal man works like an almost saturated system, in the sense that a very low stimulus induces cerebral excitation enough to perceive not only the simplest sensory functions but also the most complex ones in a synchronized way.

In relation to the scheme of cerebral gradients, and for a sensory system, the functional gradient in normal man must be understood as an ensemble of several functional densities $f_{i}$ for different qualities in gradation through the cortex, each one with different slope according to its excitability and integration demand. In the new equilibrium achieved in central syndrome the deficit of neural excitation affects these functions $f_{i}$ differently. Their incomplete cerebral integration gives place to the respective reduced sensory qualities $F_{i}$, each one reduced differently (allometrically). The mentioned disgregation phenomenon of the perception in the functional depression corresponds then to different stages of incomplete integration in different degree for the different qualities.

\section{SENSORY GROWTH, MULTISENSORY EFFECTS AND MORE SCALING POWER LAWS}

In a reduced cerebral system as was described above, the sensory level may grow by intensifying the stimulus (it includes iterative temporal summation) and by sensory facilitation by adding other different stimuli, as was said in the first section. These mechanisms involve an increase of central nervous excitation being able to com- 
pensate in part for the neural mass lost. This capability to improve the perception is greater as the magnitude of the central lesion is greater, and is null or very low in a normal man. The best example is the extreme case M, who could approach the physiological and sensory level of the less intense case $\mathrm{T}$, by the use of strong muscular stress ( $\mathrm{M}$ facilitated) as shown in Fig. 2. The inactive state, free of facilitation is difficult to achieve in acute cases as $\mathrm{M}$ since mere postural changes or weak stimuli modify the perception.

Concerning the effect of the intensity of the stimulus, Fig. 8 shows some examples of visual functions or qualities versus intensity of stimulation in a stationary regime [16, 18]. The well-known Stevens law relating perception $P$ and stimulus $S$ by a power law of the type

$$
P=p S^{m}
$$

(considered an improvement of the Fechner law) was used to fit the data, the slope of the straight line in a log-log representation being the exponent $m$. Fig. 8 (a) shows the visual field amplitude of right eye in cases $M, M$ facilitated by strong muscular stress $(40 \mathrm{~kg}$ held in his hands), and case $\mathrm{T}$, versus the illumination of a test object. As seen, the data fit rather well to Stevens straight lines, not very close of the highest values. The slope $m$ of the fitting straight lines is remarkably close to $1 / 4$ for $\mathrm{M}$ and $\mathrm{M}$ facilitated, and 1/8 for T. In Fig. $8(\mathrm{~b})$, similar representation is shown for the visual acuity in central vision, including a normal case. Straight lines with slope $1 / 4$ fit rather well to the data of case $\mathrm{M}$ inactive, the central range of the data for $\mathrm{M}$ facilitated and $\mathrm{T}$; and with slope $1 / 8$ for a normal case. In Fig. 8 (c), it is shown in linear scale representation, how the perceived direction of an upright test white arrow tends to the normal value (upright direction) as the illumination of the arrow increases, for $\mathrm{M}$ inactive and facilitated. These data do not show an admissible agreement with a simple power law and the curves merely join the experimental points.

Concerning facilitation, its effect is shown in Fig. 9, in log-log representation. Now the intensity of light on the test object was kept constant and low, say $s$. The data correspond also to a stationary regime [16, 18]. Fig. 9(a) shows the visual field amplitude of right eye in case $\mathrm{M}$, versus facilitation by muscular stress holding in his hands increasing weights. The data fit to straight lines with slopes (Stevens' powers) $1 / 2$ and $1 / 3$ for the two different diameters of the white circular test object. A greater test object implies a greater stimulus, and we can see that it leads to a lower slope, i.e., to a lower effect of the facilitation. Fig. 9 (b) shows data under similar conditions as in Fig. 9 (a) but the sensory function measured is the recovery of the upright direction $\left(180^{\circ}\right)$ of an upright test white arrow that the patient perceived tilted or almost inverted $\left(0^{\circ}\right)$ under low illumination. The data fit to power $1 / 4$. The novelty in Fig. 9(c) is that facilitation is supplied by illuminating the left eye which is not observing the object, the power of the fitting being $1 / 8$ [26]. Note that the fittings of facilitation are much better
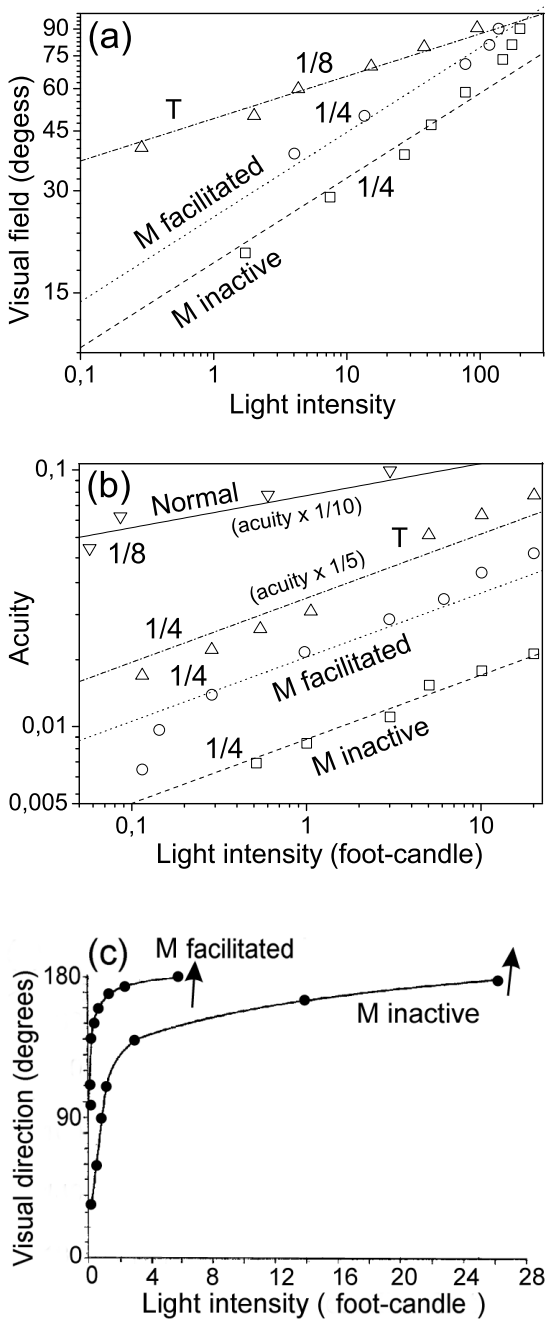

Figura 8: (a) Visual field of right eye versus relative illumination (test object: $1 \mathrm{~cm}$ diameter, white disk). $\mathrm{M}(m \approx 1 / 4)$, $\mathrm{M}$ facilitated $(m \approx 1 / 4), \mathrm{T}(m \approx 1 / 8)$. (b) Acuity of right eye versus illumination. $\mathrm{M}(m \approx 1 / 4), \mathrm{M}$ facilitated $(m \approx 1 / 4), \mathrm{T}$ $(m \approx 1 / 4)$ and normal man $(m \approx 1 / 8)$. (Adapted from Fig. $1(\mathrm{a})(\mathrm{b})$ of [26] with permission of Springer Science and Business Media). (c) Visual direction function versus illumination of an upright test arrow. (Adapted from Fog. 3(c) of [20] with permission of The MIT Press).

than those of simple stimulation.

The sensory growth by facilitation through other stimuli is a multisensory or cross-modal effect in the cerebral integrative process. Along all the cases reported in the literature on reversal of vision 25], there are some of them in which the image was reinverted by multisensory facilitation (e.g., [36, 38]). Facilitation by multisensory or cross-modal effects have been also observed in patients with visual deficits, e.g. [7, 9], and also in normals, e.g. [1, 2, 6, 44, 45, 46, 47].

The capability to improve the perception in central syndrome by multisensory facilitation was found to be greater as the deficit of excitability in the reduced ce- 

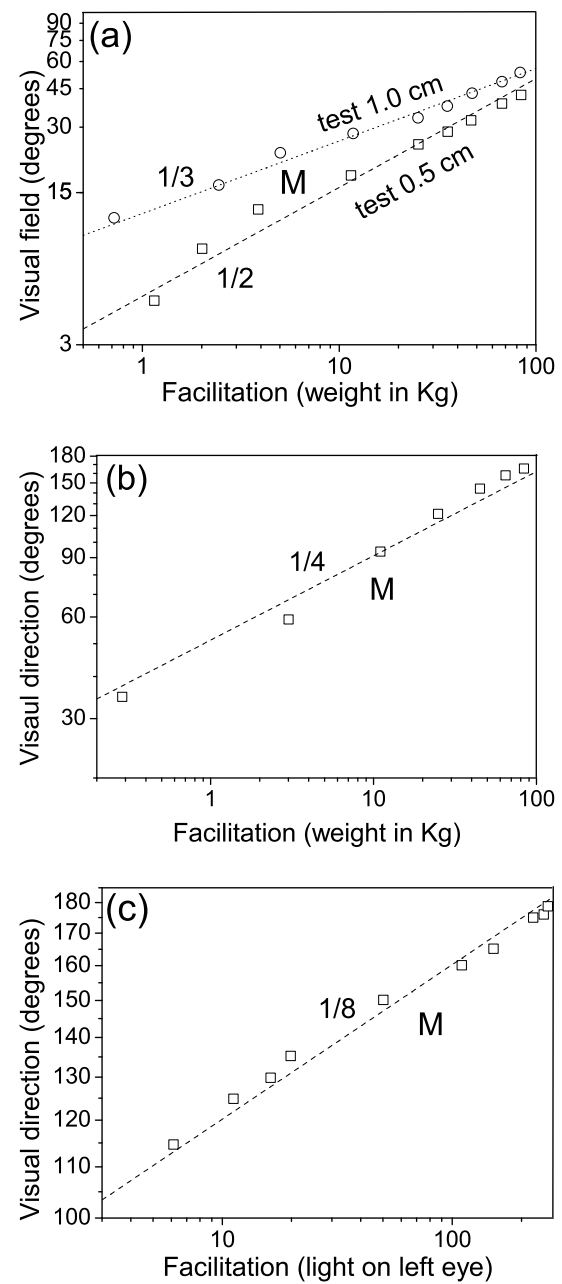

Figura 9: Case M, right eye. (a) Log-log representation of visual field amplitude versus facilitation by muscular stress holding weight in his hands. Squares: $0.5 \mathrm{~cm}$ diameter test size $(m \approx 1 / 2)$. Circles: $1.0 \mathrm{~cm}$ diameter test size $(m \approx 1 / 3)$. (b) Visual direction (reinversion) versus facilitation by muscular stress $(m \approx 1 / 4)$. (c) Visual direction versus facilitation by illumination on left eye $(m \approx 1 / 8)$. (From the respective Figs. 2(a), 2(b) and 3 of [26], with permission of Springer Science and Business Media).

rebral system is greater, and as the primary stimulus (e.g., the constant value of light $s$ and the size of the test object) is weaker [16] (see Fig. 9(a)). The first of these conditions is in relation to what is observed recently [7, 48, 49], and the last condition is also in agreement with recent observations [2, 47]. It was suggested 24] that the multisensory interaction in central syndrome leads to a nonlinear contribution to the cerebral excitation with stimuli, this being also in relation to recent suggestions [50].

For the Stevens power laws, note that their validity is assumed to be restricted to limited ranges of stimuli, and they are not exempt from criticism. However, in connection with these laws, it is remarkable that many biological observable quantities are statistically seen to scale with the mass $M$ of the organism, according to power laws $Y=k M^{r}$, where most of the exponents $r$ are multiples of $1 / 4$. These scaling laws are supposed to arise from universal mechanisms in all biological systems (in our case, neural networks) as the optimization to regulate the activity of its subunits, as cells [51, 52]. Under the assumption that a stimulus $S$ activates a neural mass $M_{\text {neur }}=\alpha S^{\beta}$ [26, 53], the above power law of perception (2) becomes $P=k\left(M_{\text {neur }}\right)^{\beta / m}=k\left(M_{\text {neur }}\right)^{r}$, i.e., we recover a biological scaling power law of growth which would be the basis of the perception laws shown above. In cases where $\beta$ would be close to unity [53], then $r \approx m$, and Stevens laws would exhibit quarter powers as seen in some of the cases here analyzed.

\section{CONCLUSIONS}

The functional model described highlights a functional unity and continuity of the cerebral cortex. This is reflected in the functional gradients according to which the specificity of the cortex is distributed with a continuous variation, giving place to regions where several specificities overlap significantly. A functional unity is suggested in the sense that for a sensory function to be normal, the integrative process must involve the whole specific gradient extended over the cortex and not only over the region with higher functional density. Multisensory integration would be involved in a greater degree in regions where the overlapping of the specific functional gradients is greater. This scheme affords an interpretation of the great variety of syndromes and responds to requirements formulated recently, as said in the introduction.

A neural mass lost in the rather unspecific region where the decline of several gradients overlap, as in the so-called central zone, is interpreted as a scale reduction in the nervous excitability of the cerebral system. The functional gradients associated to the different sensory qualities of a given sensory system become then affected allometricaly, leading to stages in perception of incomplete integration in a different degree for each quality. Inverted or tilted perception appears as an stage of incomplete integration. The higher or complex functions, which require greater nervous excitation (and then integration), are more affected and lost first. A functional continuity is manifested from elementary sensory functions to higher ones, according to their excitability thresholds, following a physiological order, as is shown in the dynamic disgregation of qualities as excitation diminishes. Allometric scaling laws account for the continuous variation of each quality (e.g., visual direction, acuity) in relation to another quality (e.g., visual field).

A growth in the sensory level, and then a more complete integration, is achieved by means of an increase in the primary stimulus or by multisensory facilitation, mechanisms which supply in part the neural mass lost. This capability is greater as the deficit is greater and the 
primary stimulus is weaker. The growth of the sensory level follows approximately scaling power laws that would emerge from the dynamics of biological neural networks.

Note that the approach exposed could be connected with those based on a distributed character of the cere- bral processing and its adaptive and long-distance integrative aspects (e.g, [54, 55]).

Acknowledgement. I am very grateful to M.A. Porras for his valuable assistance in preparing this manuscript.
[1] R. Martuzzi, M.M.Murray, C.M. Michel et al., Multisensory interactions within human primary cortices revealed by BOLD dynamics, Cerb. Cortex 17 (2007) 1672-1679.

[2] H. Gillmeister and M. Eimer, Tactile enhancement of auditory detection and perceived loudness, Brain Res. 1160 (2007) 58-68.

[3] C. Kayser and N.K. Logothetis, Do early sensory cortices integrate cross-modal information?, Brain Struct. Funct. 212 (2007) 121-132.

[4] C. Kayser, C.I. Petkov, M. Augath and N.K. Logothetis, Functional Imaging reveals visual modulation of specific fields in auditory cortex, J. Neurosci. 27 (2007) 18241835.

[5] J.C. Alvarado, J.W. Vaughan, T.R. Stanford and B.E. Stein, Multisensory versus Unisensory Integration: Contrasting Modes in the Superior Colliculus, J. Neurophysiol. 27 (2007) 1824-1835.

[6] A. Diederich and H. Colonius, Why two "Distractors. ${ }^{a}$ re better than one: modeling the effect of non-target auditory and tactile stimuli on visual saccadic reaction time, Ex. Brain Res. 179(2007) 43-54.

[7] D.A. Poggel, E. Kasten, E.M. Muller-Oehring et al., Improving residual vision by attentional cueing in patients with brain lesions, Brain Res. 1097 (2006) 142-148.

[8] J.K. Bizley, F.R. Nodal, V.M. Bajo et al., Physiological and Anatomical Evidence for Multisensory Interactions in Auditory Cortex, Cereb. Cortex 17 (2007)2172-2189.

[9] F. Frassinetti, N. Bolognini, D. Bottari et al., Audiovisual integration in patients with visual deficit, J. Cogn. Neurosci. 17 (2005) 1442-1452.

[10] E. Macaluso, C.D. Frith, J. Driver, Multisensory stimulation with or without saccades: fMRI evidence for crossmodal effects on sensory-specific cortices that reflect multisensory location-congruence rather than task-relevance, Meuroimage 26 (2005) 414-425.

[11] H. Théoret, L. Merabet, A. Pascual-Leone, Behavioral and neuroplastic changes in the blind: evidence for functionally relevant cross-modal interactions, J. Physiol. Paris. 98 (2004) 221-33.

[12] G.A. Calvert and T. Thesen, Multisensory integration: methodological approaches and emerging principles in the human brain, J. Physiol. Paris 98 (2004) 191-205.

[13] K. Sathian and A.Zangaladze, Feeling with the mind's eye: contribution of visual cortex to tactile perception, Behav. Brain Res. 135 (2002) 127-132.

[14] L.G. Cohen, P. Celnik, A. Pascual-Leone et al., Functional relevance of cross-modal plasticity in blind humans, Nature 389 (1997) 180-3.

[15] M.T. Wallace, R. Ramachandran and B.E. Stein, A revise view of sensory cortical parcellation, Proc. Natl. Acad. Sci. USA 101 (2004) 2167-2172.

[16] J. Gonzalo, Investigaciones sobre la nueva dinámica cerebral. La actividad cerebral en función de las condiciones dinámicas de la excitabilidad nerviosa (Publicaciones del
Consejo Superior de Investigaciones Científicas, Inst. S. Ramón y Cajal, Madrid, Vol. I 1945, Vol. II 1950). (Avalable in: Instituto Cajal, CSIC, Madrid).

[17] J. Gonzalo, La cerebración sensorial y el desarrollo en espiral. Cruzamientos, magnificación, morfogénesis, Trab. Inst. Cajal Invest. Biol. 43 (1951) 209-260.

[18] J. Gonzalo, Las funciones cerebrales humanas según nuevos datos y bases fisiológicas: Una introducción a los estudios de Dinámica Cerebral, Trab. Inst. Cajal Invest. Biol. 44 (1952) 195-157.

[19] K. Goldstein and A. Gelb, Psychologische Analysen hirnpathologischer Fälle auf Grund Untersuchungen Hirnverletzer, Zeitschrift für die gesamte Neurologie und Psychiatrie 41 (1918) 1-142.

[20] I. Gonzalo and A. Gonzalo, Functional gradients in cerebral dynamics: The J. Gonzalo theories of the sensorial cortex, in: R. Moreno-Díaz and J.Mira, eds., Brain Processes, Theories and Models. An Int. Conf. in honor of W.S. McCulloch 25 years after his death (The MIT Press, Massachusetts 1996) 78-87.

[21] I. Gonzalo, Allometry in the J. Gonzalo's model of the sensorial cortex, Lect. Not. Comp. Sci. 1240 (1997) 169177.

[22] I. Gonzalo, Spatial Inversion and Facilitation in the J. Gonzalo's Research of the Sensorial Cortex. Integrative Aspects, Lect. Not. Comp. Sci. 1606 (1999) 94-103.

[23] I. Gonzalo and M.A. Porras, Time-dispersive effects in the J. Gonzalo's research on cerebral dynamics, Lect. Not. Comp. Sci. 2084 (2001) 150-157.

[24] I. Gonzalo and M.A. Porras, Intersensorial summation as a nonlinear contribution to cerebral excitation, Lect. Not. Comp. Sci. 2686 (2003) 94-101.

[25] I. Gonzalo-Fonrodona, Inverted or tilted inversion disorder, Rev. Neurol. 44 (2007) 157-165.

[26] I. Gonzalo-Fonrodona and M.A. Porras, Physiological Laws of Sensory Visual System in Relation to Scaling Power Laws in Biological Neural Networks, Lect. Not. Comp. Sci. 4527 (2007) 96-102.

[27] M Critchley, The Parietal Lobes (Arnold, London 1953) Chap 9.

[28] M.B. Bender and H.L. Teuber, Neuro-ophthalmology, Prog. Neurol. Psychiatry III (1948) 163-182.

[29] J.de Ajuriaguerra J and H. Hécaen, Le Cortex Cérébral Étude Neuro-psycho-pathologique (Masson, Paris 1949).

[30] A.E. Delgado, Modelos Neurocibernéticos de Dinámica Cerebral. Ph.D.Thesis, E.T.S. de Ingenieros de Telecomunicación, Universidad Politécnica de Madrid, 1978.

[31] J. Mira, A.E. Delgado and R. Moreno-Díaz, The fuzzy paradigm for knowledge representation in cerebral dynamics, Fuzzy Sets and Systems 23 (1987) 315-330.

[32] J. Mira, A. Manjarrés, S. Ros et al., Cooperative Organization of Connectivity Patterns and Receptive Fields in the Visual Pathway: Application to Adaptive Thresholdig, Lect. Not. Comp. Sci. 930 (1995) 15-23. 
[33] A. Manjarrés, Modelado Computacional de la Decisión Cooperativa: Perspectivas Simbólica y Conexionista. Ph.D. Thesis, Ciencias Físicas, Facultad de Ciencias de la UNED, Madrid, 2001.

[34] M. Arias and I. Gonzalo, La obra neurocientífica de Justo Gonzalo (1910-1986): El síndrome central y la metamorfopsia invertida, Neurología 19 (2004) 429-433.

[35] L. Barraquer, La dinámica cerebral de Justo Gonzalo en la historia, Neurología 20 (2005) 169-173.

[36] Y. River, T. Ben Hur and I. Steiner, Reversal of vision metamorphopsia, Arch. Neurol. 53 (1998) 1362-1368.

[37] M. Arias, C. Lema, I. Requena et al., Metamorfopsia invertida: una alteración en la percepción de la situación espacial de los objetos, Neurología 16 (2001) 149-153.

[38] A. Arjona and E. Fernández-Romero, Ilusión de inclinación de la imagen visual. Descripción de dos casos y revisión de la terminología, Neurología 17 (2002) 338-341.

[39] D.D. Malis and J.P. Guyot, Room tilt illusion as a manifestation of peripheral vestibular disorders, Ann. Otol. Rhinol. Laryngol. 112 (2003) 600-605.

[40] A. H. Hernández, F. Pujadas, F. Purroy et al., Upside down reversal of vision due to an isolated acute cerebellar ischemic infarction, J. Neurol. 253 (2006) 953-954.

[41] A. Unal, A. Cila and S. Saygi, Reversal of vision metamorphopsia: A manifestation of focal seizure due to cortical dysplasia, Epilepsy Behav. 8 (2006) 308-311.

[42] E. Kasten, and D.A. Poggel, A Mirror in the Mind: A Case of Visual Allaesthesia in Homonymous Hemianopia, Neurocase 12 (2006) 98-106.

[43] J. Perkkiö and R. Keskinen, The relationship between growth and allometry, J. theor. Biol. 113 (1985) 81-87.

[44] N.P. Holmes, G.A. Calvert and C. Spence, Tool use changes multisensory interactions in seconds: evidence from crossmodal congruency task. Exp. Brain Res. 183 (2007) 465-476.

[45] B.A. Rowland, S. Quessy, T.R. Standford and B.E. Stein, Multisensory integration shortens physiological latencies,
J. Neurosc. 27 (2007) 5879-5884.

[46] B. Baier, A. Kleinschmidt and N.G. Müller, Cross-modal processing in early visual and auditory cortices depends on expected statistical relationship of multisensory information, J. Neurosci. 26 (2006) 12260-12265.

[47] J.W. Schnupp, K.L. Dawe and G.L. Pollack, The detection of multisensory stimuli in an orthogonal sensory space, Exp. Brain Res. 162 (2005) 181-190.

[48] P.J. Laurienti, J.H. Burdette, J.A. Maldjian and M.T. Wallace, Enhanced multisensory integration in older adults, Neurobiol Aging 27 (2006) 1155-1163.

[49] A.M. Peiffer, J.L. Mozolic, C.E. Higenschmidt and P.J. Laurienti, Age-related multisensory enhancement in a simple audiovisual detection task, Neuroreport 18 (2007) 1077-1081.

[50] T.R. Standford and B.E. Stein, Superadditivity in multisensory integration: putting the computation in context, Neuroreport 18 (2007) 787-792.

[51] G.B. West and J.H. Brown, The origin of allometric scaling laws in biology from genomes to ecosystems: towards a quantitative unifying theory of biological structure and organization, J. Exper. Biol. 208 (2005) 1575-1592.

[52] R.B. Anderson, The power law as an emergent property, Mem. Cogn. 29 (2001) 1061-1068.

[53] O.J. Arthurs, C.M.E. Stephenson, K. Rice et al., Dopaminergic effects on electrophysiological and functional MRI measures of human cortical stimulus-response power laws, NeuroImage 21 (2004) 540-546.

[54] S. Yuval-Greenberg, L. Deouell, What You See Is Not (Always) What You Hear: Induced Gamma Band Responses Reflect Cross-Modal Interactions in Familiar Object Recognition, J. Neurosci. 27 (2007) 1090-1096.

[55] E. Rodríguez, N. George, J.P. Lachaux et al., Perception's shadow: long-distance synchronization of human brain activity, Nature 397 (1999) 430-433. 